\title{
Simulation of Complete Liquid-Vapor Phase Change inside Divergent Porous Evaporator
}

\author{
Omar Rafae Alomar, Miguel A. A. Mendes, Dimosthenis Trimis, and Subhashis Ray
}

\begin{abstract}
Complete phase change process within a divergent porous evaporator is numerically investigated in this paper. $\mathrm{A}$ smoothing algorithm, proposed by the present authors, is used in order to deal with the discontinuity in effective diffusion coefficient. Effects of various parameters on the temperature distribution are carefully investigated, which clearly indicate that operating conditions and the geometry of diffuser strongly influence the outlet condition of steam, whereas, porous media properties have only minor impact.
\end{abstract}

Index Terms - Complete phase change, divergent evaporator, porous media, smoothing of diffusion coefficient.

\section{INTRODUCTION}

Phase change inside porous media forms an important class of problems that finds applications in various engineering disciplines [1]. Two-phase flow within porous media has been traditionally solved by employing different idealization, e.g., separated flow model [2], [3], separated phase Model [4], [5] and Two-Phase Mixture Model (TPMM) [6]-[8]. Among them, the TPMM of [8] represents a general numerical formalism for efficient simulation of phase-change processes inside porous media. This model is characterized by the coexistence of a two-phase zone surrounded by single-phase regions with moving interfaces and being more convenient than others, is widely used for solving phase change problems within porous media. Owing mainly to this reason, several researchers [9]-[13] in the past used the model of Wang [8] for numerical simulations under different flow conditions inside porous media.

A careful review of literature reveals that all the previous studies dealt with incomplete phase change process. To the best of the present authors' knowledge, there is no study available in the open literature where complete phase change process (i.e., from a sub-cooled liquid state to the superheated vapor) inside porous media is numerically simulated. Perhaps this can be explained by the presence of discontinuity in the modeled effective diffusion coefficient close to saturated

Manuscript received November 27, 2013; revised March 20, 2014. This work was partially supported by the German Academic Exchange Service (DAAD) and the Ministry of Higher Education and Scientific Research of Iraq through their scholarship grant to O.R. Alomar.

O. R. Alomar is with the Institute of Thermal Engineering (IWTT), Technische Universität Bergakademie Freiberg (TU-BAF), Gustav-Zeuner Straße 7, 09596 Freiberg, Germany and the Technical college of Mosul, Cultural group Street, Mosul, Iraq (e-mail: omar.alomar@ iwtt.tu-freiberg.de).

M. A. A. Mendes, D. Trimis, and S. Ray are with the IWTT, TU-BAF, Gustav-Zeuner Straße 7, 09596 Freiberg, Germany (e-mail: miguel.mendes@iwtt.tu-freiberg.de, trimis@iwtt.tu-freiberg.de, ray@iwtt.tu-freiberg.de). liquid and vapor phases, which may lead to non-physical "jump" in the predicted temperature. In order to deal with such discontinuity and enable simulation of complete phase change process within porous media based on the TPMM [8], present authors proposed a smoothing algorithm for the effective diffusion coefficient [14].

Another important aspect of evaporation inside porous media is the volume expansion of phase change fluid due to huge difference in densities of liquid and vapor phases. Since the vapor-phase occupies significantly larger volume, the velocity increases considerably in a constant cross-sectional area duct during a liquid-vapor phase change process. Under this situation, it would be worthwhile to explore the possibility of using a duct with increasing cross-sectional area in the axial direction.

The aim of the present work, therefore, is to numerically investigate the complete phase change process inside a divergent porous evaporator using TPMM [8] and its modification [14] with one-dimensional formulation. In addition, the interaction between phases (liquid to two-phase and two-phase to vapor) and effects of different parameters such as porosity, outlet pipe diameter, heat flux, pipe length, length of divergent section and inlet velocity on the flow and temperature fields are also considered for simulations.

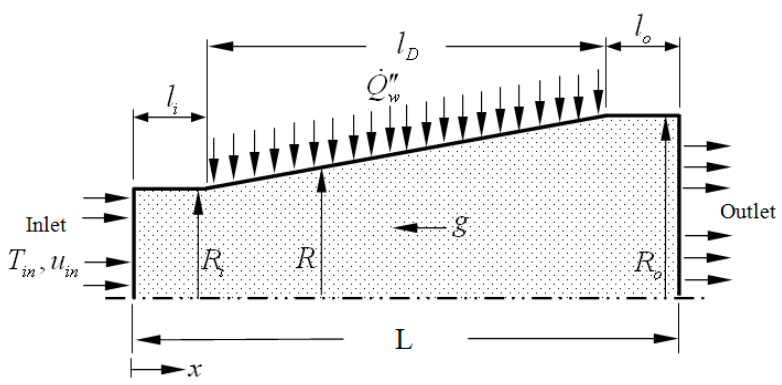

Fig. 1. Schematic representation of the phase change problem.

\section{MATHEMATICAL FORMULATION}

\section{A. Problem Description}

Schematic representation a divergent porous evaporator, considered for the simulation of complete phase change process, is shown in Fig. 1 along with its dimensions. The pipe has increasing cross-sectional area in the axial direction and is filled with an isotropic, homogenous porous medium. An external pressure gradient drives the sub-cooled liquid water, entering at a temperature $T_{\text {in }}$ (lower than the saturation temperature $T_{\text {sat }}$ ) and a velocity $u_{i n}$, to flow through the pipe. It is then heated to the superheated vapor state by applying uniform heat flux on the external surface of the evaporator at 
the diffuser section (Fig. 1). Further, the flow is considered steady as well as one dimensional and all properties are assumed to be constant for both liquid and vapor phases.

\section{B. Governing Equations and Boundary Conditions}

In the present study, all conservation equations are made dimensionless according to definitions presented in Table I, where symbols have their usual meaning. They are carefully chosen in order to retain all governing equations along with expressions for mixture variables in the same form as in [8]. Integrating dimensionless forms of the resultant governing equations over the entire pipe cross-section at a given axial location, the respective one-dimensional forms can be obtained, where variations remain only in the axial direction. Thus, for a steady state problem, the mass conservation equation can be written as:

$$
\frac{d}{d x^{*}}\left(\rho^{*} u_{x}^{*} A_{c}^{*}\right)=0
$$

where symbols with superscripts '*' denote dimensionless values, $A_{c}^{*}=R^{* 2} / 2$ is the pipe cross-sectional area per unit radian and $R^{*}$ is the local pipe radius. Conservation of energy, on the other hand, is obtained as:

$$
\begin{aligned}
& \frac{d}{d x^{*}}\left(A_{c}^{*} \gamma_{h} u_{x}^{*} H^{*}\right)=\frac{d}{d x^{*}}\left(A_{c}^{*} \Gamma_{h}^{*} \frac{d H^{*}}{d x^{*}}\right) \\
& +\frac{d}{d x^{*}}\left(A_{c}^{*} f \frac{K^{*} \Delta \rho^{*} h_{f g}^{*}}{v_{v}^{*}} b_{x}^{*}\right)+P^{*} \dot{Q}_{w}^{\prime *}
\end{aligned}
$$

with $P^{*}=R^{*}$ being the pipe perimeter and $b_{x}^{*}$ is the body force per unit mass in the $x$-direction. In Table I, $\tilde{b}_{x}=b_{x} / g$ represents the normalized body force with respect to acceleration due to gravity $g$ and $F r=u_{i n} / \sqrt{g R_{i}}$ is the Froude number. In the definition of viscosity, $R e_{l}=\rho_{l} u_{i n} R_{i} / \mu_{l}$ is the inlet Reynolds number and $v_{v}^{*}=\mu_{v}^{*} / \rho_{v}^{*}$ in (2) is the kinematic viscosity of the vapor phase. Further definitions of mixture properties, appearing in (1) and (2), are listed in Tables I and II, where $s$ is the liquid volume fraction and subscripts ' $l$ ', ' $v$ ' and ' $s$ ' stand for liquid, vapor (for fluid) and solid (for porous medium) phases, respectively. The expression for effective diffusion coefficient $\Gamma_{h}^{*}$ in (2) contains the effective thermal conductivity $k_{\text {eff }}$ in its dimensionless form, which in the present investigation is obtained from the parallel arrangement model. As shown in Table I, the dimensionless thermal conductivity of liquid phase is given as $k_{l}^{*}=1 / P e_{l}$, where $P e_{l}=u_{i n} R_{i} / \alpha_{l}$ is the Peclet number based on velocity and pipe radius at the inlet and liquid properties. It may be further noted that Reynolds and Peclet numbers are related to each other as $P e_{l}=R e_{l} P r_{l}$, where $P r_{l}=v_{l} / \alpha_{l}$ is the Prandtl number of the liquid phase. Therefore, $P e_{l}$ is implicitly specified by fixing $R e_{l}$ and the working fluid, i.e., $P r_{l}$.

Evaluation of quantities, listed in Table II, requires the liquid saturation $s$, which, along with the local temperature $T^{*}$, can be calculated from the enthalpy as shown in Table III.
TABLE I: DEFINITIONS OF DIMENSIONLESS VARIABLES

\begin{tabular}{|c|c|}
\hline Variables & Expressions \\
\hline Density & $\rho^{*}=\rho_{l}^{*} s+\rho_{v}^{*}(1-s)$ \\
\hline Mass velocity & $\rho^{*} \mathbf{u}^{*}=\rho_{l}^{*} \mathbf{u}_{l}^{*}+\rho_{v}^{*} \mathbf{u}_{v}^{*}$ \\
\hline Enthalpy & $\rho^{*} h^{*}=h_{l}^{*} \rho_{l}^{*} s+(1-s) \rho_{v}^{*} h_{v}^{*}$ \\
\hline Kinematic viscosity & $v^{*}=\left[k_{r l} / v_{l}^{*}+k_{r v} / v_{v}^{*}\right]^{-1}$ \\
\hline $\begin{array}{l}\text { Effective thermal } \\
\text { conductivity }\end{array}$ & $k_{e f f}^{*}=(1-\varepsilon) k_{s}^{*}+\varepsilon\left\lfloor k_{l}^{*} s+(1-s) k_{v}^{*}\right\rfloor$ \\
\hline $\begin{array}{l}\text { Advection correction } \\
\text { coefficient }\end{array}$ & $\gamma_{h}=\frac{\left[s+\left(\rho_{v}^{*} / \rho_{l}^{*}\right)(1-s)\right]\left[h_{v, s a t}^{*}\left(1+\lambda_{l}\right)-h_{l, s a t}^{*} \lambda_{l}\right]}{\left(2 h_{v, s a t}^{*}-h_{l, s a t}^{*}\right) s+(1-s)\left(\rho_{v}^{*} h_{v, s a t}^{*} / \rho_{l}^{*}\right)}$ \\
\hline $\begin{array}{l}\text { Effective diffusion } \\
\text { Coefficient (diffusivity) }\end{array}$ & $\Gamma_{h}^{*}=\frac{D^{*} h_{f g}^{*}}{\rho_{l}^{*} h_{f g}^{*}+\left(\rho_{l}^{*}-\rho_{v}^{*}\right) h_{v, s a t}^{*}}+k_{e f f}^{*} \frac{d T^{*}}{d H^{*}}$ \\
\hline $\begin{array}{l}\text { Capillary diffusion } \\
\text { coefficient }\end{array}$ & $D^{*}=\frac{\left(\varepsilon K^{*}\right)^{1 / 2} \sigma^{*}}{v_{l}^{*}} \frac{k_{r v} k_{r l}}{\left(v_{v}^{*} / v_{l}^{*}\right) k_{r l}+k_{r v}}\left(-\frac{d J}{d s}\right)$ \\
\hline $\begin{array}{l}\text { Surface tension } \\
\text { coefficient }\end{array}$ & $\sigma^{*}=\sigma / \rho_{l} u_{i n}^{2} R_{i}$ \\
\hline Relative mobilities & $\lambda_{l}=\frac{k_{r l} / v_{l}^{*}}{k_{r l} / v_{l}^{*}+k_{r v} / v_{v}^{*}} ; \lambda_{v}=\frac{k_{r v} / v_{v}^{*}}{k_{r l} / v_{l}^{*}+k_{r v} / v_{v l}^{*}}$ \\
\hline Hindrance function & $f=k_{r v} \lambda_{l}=\frac{k_{r l} k_{r v} / v_{l}^{*}}{k_{r l} / v_{l}^{*}+k_{r v} / v_{v}^{*}}$ \\
\hline Relative permeabilities & $k_{r l}=s^{n} ; k_{r v}=(1-s)^{n} ; n=3$ \\
\hline $\begin{array}{l}\text { Capillary pressure } \\
\text { function }\end{array}$ & $J=1.417(1-s)-2.120(1-s)^{2}+1.263(1-s)^{3}$ \\
\hline
\end{tabular}

\begin{tabular}{ll}
\hline \hline Dimensionless variables & Expressions \\
\hline Length & $x^{*}=x / R_{i} ; r^{*}=r / R_{i}$ \\
Density & $\rho^{*}=\rho / \rho_{l} ; \rho_{l}^{*}=1$ \\
Velocity & $u^{*}=u / u_{i n}$ \\
Temperature & $T^{*}=T C_{p l} / h_{f g}$ \\
Enthalpy & $h^{*}=h / h_{f g} ; h_{f g}^{*}=1$ \\
Modified volumetric enthalpy & $H^{*}=H / \rho_{l} h_{f g}=\rho^{*}\left(h^{*}-2 h_{v, s a t}^{*}\right)$ \\
Specific heat & $C_{p}^{*}=C_{p} / C_{p l} ; C_{p l}^{*}=1$ \\
Dynamic viscosity & $\mu^{*}=\mu / \mu_{l} R e_{l} ; \mu_{l}^{*}=1 / R e_{l}$ \\
Thermal conductivity & $k^{*}=k / \rho_{l} C_{p l} R_{i} u_{i n}=k / k_{l} P e_{l}$ \\
Body force per unit mass & $b_{x}^{*}=\tilde{b}_{x} g R_{i} / u_{i n}^{2}=\tilde{b}_{x} / F r^{2}$ \\
Heat flux & $\dot{Q}_{w}^{*}=\dot{Q}_{w}^{\prime \prime} / \rho_{l} u_{i n} h_{f g}$ \\
Permeability or Darcy number & $K^{*}=K / R_{i}^{2}$ \\
\hline \hline
\end{tabular}

TABLE II: FURTHER DEFINITIONS OF DIMENSIONLESS MIXTURE PROPERTIES

TABLE III: SUPPLEMENTARY RELATIONS BETWEEN MODIFIED VOLUMETRIC ENTHALPY, TEMPERATURE, AND LIQUID SATURATION

\begin{tabular}{cccc}
\hline \hline$H^{*}$ & $T^{*}$ & $s$ & $d T^{*} / d H^{*}$ \\
\hline$H^{*} \leq H_{l, s a t}^{*}$ & $\frac{H^{*}+2 \rho_{l}^{*} h_{v, s a t}^{*}}{\rho_{l}^{*} C_{p l}^{*}}$ & 1 & $1 / \rho_{l}^{*} C_{p l}^{*}$ \\
$H_{l, s a t}^{*}<H^{*} \leq H_{v, s a t}^{*}$ & $T_{s a t}^{*}$ & $-\frac{H^{*}+\rho_{v}^{*} h_{v, s a t}^{*}}{1+\left(\rho_{l}^{*}-\rho_{v}^{*}\right) h_{v, s a t}^{*}}$ & 0 \\
$H_{v, s a t}^{*}<H^{*}$ & $T_{s a t}^{*}+\frac{H^{*}+\rho_{v}^{*} h_{v, s a t}^{*}}{\rho_{v}^{*} C_{p v}^{*}}$ & 0 & $1 / \rho_{v}^{*} C_{p v}^{*}$ \\
\hline \hline$H_{l, s a t}^{*}=-\rho_{l}^{*}\left(2 h_{v, s a t}^{*}-h_{l, s a t}^{*}\right)=2 h_{v, s a t}^{*}-h_{l, s a t}^{*} ; H_{v, s a t}^{*}=-\rho_{v}^{*} h_{v, s a t}^{*}$ &
\end{tabular}

Solution of (1) and (2) requires appropriate (as well as consistent) boundary conditions. At the inlet, i.e., at $x^{*}=0$, $u_{x}^{*}=1$ and $H^{*}=\rho_{i n}^{*}\left(C_{p l}^{*} T_{i n}^{*}-2 h_{v, s a t}^{*}\right)$ are prescribed. Further, since sub-cooled liquid enters the pipe, $\rho_{i n}^{*}=1$ is set. On the other hand, at the outlet, i.e., at $x^{*}=L / R_{i}$, the second derivatives of all variables are set to zero.

Under steady-state condition, owing to the conservation of mass in (1), the mass flow rates at all the cell faces can be set 
equal to that at the inlet and hence they are calculated as:

$$
\dot{m}_{c f}^{*}=\dot{m}_{i n}^{*}=\rho_{c f}^{*} u_{c f}^{*} A_{c}^{*}
$$

where the suffix ' $c f$ ' stands for cell faces and the pressure variation does not affect the velocity solution. Since by definition, the dimensionless density and velocity at the inlet are both set equal to unity, the cell face velocity can be directly obtained from (3) as: $u_{c f}^{*}=A_{c, i n}^{*} /\left(\rho_{c f}^{*} A_{c}^{*}\right)$, where $\rho_{c f}^{*}$ has to be calculated iteratively from the known liquid saturation as shown in Table II. The outlet volumetric enthalpy $H^{*}$, however, is required for solving (2), which is obtained by linear extrapolation using values at the neighboring interior nodes that ensures $\partial^{2} H^{*} / \partial x^{* 2}=0$ at the outlet.

\section{NUMERICAL SOLUTION}

\section{A. Discretization of Energy Equation}

The discretization of energy conservation (2) is carried out by the Finite Volume Method (FVM) [15]. The fist-order accurate Upwind Differencing Scheme (UDS) is employed for expressing the convective term, whereas the second order accurate Central Differencing Scheme (CDS) is used in order to deal with the diffusive term. Effective diffusion coefficients and advection correction coefficients at cell faces are obtained using the harmonic mean approximation [15] (that ensures the balance of diffusive energy flux) and the linear interpolation from the adjacent nodal values, respectively. Owing to the strong non-linearity in (2), arising out of the interdependence of dependent variable $H^{*}$, mixture properties and axial velocity, discretized equations are further under-relaxed and an under-relaxation factor of 0.1 is generally used for most of the simulations in order to achieve convergence. The resulting tri-diagonal system of simultaneous equations is solved iteratively using the Thomas algorithm [15]. For all the cases presented in this article, the convergence criterion is set to $10^{-5}$, which ensures the overall energy conservation in the system to be satisfied within $0.01 \%$.

\section{B. Treatment of Diffusion Coefficient}

According to the experience of the present authors, the major problem that is encountered while modeling the complete phase change process within a porous medium is the treatment of discontinuities in the effective diffusion coefficient at the boundaries of two-phase regions (i.e., between sub-cooled liquid and two-phase as well as two-phase and superheated vapor). It may be noted from Table II that $\Gamma_{h}^{*}$ has two distinct parts. In the single phase regions, $D^{*}=0$ (since either $k_{r v}=0$ or $k_{r l}=0$ ), whereas in the two-phase region, $d T^{*} / d H^{*}=0$ (see Table III). Therefore, for $s=0$ (saturated vapor) and $s=1$ (saturated liquid), those uniquely identify the exact physical conditions (states) of the working fluid, two different values of $\Gamma_{h}^{*}$ are obtained with one of them, calculated from the two-phase region, being exactly equal to zero as both $D^{*}$ and $d T^{*} / d H^{*}$ are zero for these conditions. As it will be shortly apparent, such discontinuities, under certain conditions, can produce non-physical "jump" in the predicted temperature. In order to eliminate these discontinuities, smoothing functions for $\Gamma_{h}^{*}$ are applied, following the suggestion of Alomar et al. [14], for the following four regions: i) sub-cooled liquid phase, close to $T=T_{\text {sat }}$, ii) superheated vapor phase, close to $T=T_{\text {sat }}$, iii) two-phase region, close to $s=1$ and iv) two-phase region, close to $s=0$. The overall smoothing algorithm is briefly described next in this section.

For the single phase regions i) and ii), $\Gamma_{h}^{*}$ is expressed as:

$$
\begin{aligned}
& \Gamma_{h}^{*}=\left[\left(\Gamma_{h l}^{*}\right)^{-m_{\phi l}}+\left(\Gamma_{h \phi, l}^{*}\right)^{-m_{\phi l}}\right]^{-1 / m_{\phi l}} \\
& \Gamma_{h}^{*}=\left[\left(\Gamma_{h v}^{*}\right)^{-m_{\phi v}}+\left(\Gamma_{h \phi, v}^{*}\right)^{-m_{\phi \nu}}\right]^{-1 / m_{\phi v}},
\end{aligned}
$$

where, $m_{\phi l}$ and $m_{\phi v}$ are the exponents for asymptotes, suggested by Churchill and Usagi [16], whereas $\Gamma_{h \phi, l}^{*}$ and $\Gamma_{h \phi, v}^{*}$ are given as:

$$
\begin{aligned}
\Gamma_{h l, \phi}^{*} & =\Gamma_{h l}^{*}\left[\psi_{l}+\phi_{l}\left(1-\psi_{l}\right)\right] \\
\Gamma_{h v, \phi}^{*} & =\Gamma_{h v}^{*}\left[\psi_{v}+\phi_{v}\left(1-\psi_{v}\right)\right] .
\end{aligned}
$$

In (5), $\Gamma_{h}^{*}$ is assigned values equal to $\psi_{l} \Gamma_{h l}^{*}$ and $\psi_{v} \Gamma_{h v}^{*}$ for saturated liquid and vapor states, respectively. Further, $\phi_{l}$ and $\phi_{v}$ are scaled temperatures for liquid and vapor phases, respectively, and are defined as:

$$
\begin{aligned}
\phi_{l} & =\left(T_{\text {sat }}^{*}-T^{*}\right) / \Delta T_{l}^{*} \\
\phi_{v} & =\left(T^{*}-T_{\text {sat }}^{*}\right) / \Delta T_{v}^{*},
\end{aligned}
$$

where, $\Delta T_{l}^{*}$ and $\Delta T_{v}^{*}$ are the temperatures over which $\Gamma_{h}^{*}$ is relaxed in liquid and vapor phases, respectively. In (4)-(6), $m_{\phi l}, m_{\phi v}, \psi_{l}, \psi_{v}, \Delta T_{l}^{*}$ and $\Delta T_{v}^{*}$ are adjustable parameters to be chosen according to the requirement.

Analogously for the two-phase regions iii) and iv), $\Gamma_{h}^{*}$ is expressed as:

$$
\begin{gathered}
\Gamma_{h}^{*}=\left[\left(\Gamma_{h l, s}^{*}\right)^{m_{s l}}+\left(\Gamma_{h s}^{*}\right)^{m_{s l}}\right]^{1 / m_{s l}} \\
\Gamma_{h}^{*}=\left[\left(\Gamma_{h v, s}^{*}\right)^{m_{s v}}+\left(\Gamma_{h s}^{*}\right)^{m_{s v}}\right]^{1 / m_{s v}},
\end{gathered}
$$

where, $m_{s l}$ and $m_{s v}$ are similar exponents for asymptotes and $\Gamma_{h s}^{*}$ is the value of diffusion coefficient that one would obtain for a given value of $s$, whereas $\Gamma_{h l, s}^{*}$ and $\Gamma_{h v, s}^{*}$ are given as:

$$
\begin{gathered}
\log \left(\Gamma_{h l, s}^{*}\right)=\log \left(\psi_{l} \Gamma_{h l}^{*}\right)+\frac{1-s}{1-s_{l}}\left[\log \left(\Gamma_{h, s_{l}}^{*}\right)-\log \left(\psi_{l} \Gamma_{h l}^{*}\right)\right] \\
\log \left(\Gamma_{h v, s}^{*}\right)=\log \left(\psi_{v} \Gamma_{h v}^{*}\right)+\frac{s}{s_{v}}\left[\log \left(\Gamma_{h, s_{v}}^{*}\right)-\log \left(\psi_{v} \Gamma_{h v}^{*}\right)\right]
\end{gathered}
$$

In (8), $s_{l}$ and $s_{v}$ are the cut-off liquid saturations close to saturated liquid and vapor phases, respectively, for which the 
true values of $\Gamma_{h}^{*}$ are obtained as $\Gamma_{h, s_{l}}^{*}$ and $\Gamma_{h, s_{v}}^{*}$, respectively. Quite obviously, in (7) and (8), $m_{s l}, m_{s v}, s_{l}$ and $s_{v}$ are the adjustable parameters those dictate the degree of smoothing of effective diffusion coefficient in the two-phase region.

In the present study, water is considered as the working fluid and its properties are given in Table IV, where the saturation temperature $T_{\text {sat }}$ is taken as $100^{\circ} \mathrm{C}$ [9], [17], [18]. The consequence of smoothing is demonstrated in Fig. 2, where other than the properties of water, $k_{s}=20 \mathrm{~W} / \mathrm{mK}$ is used, which is typical for compressed wire-mesh structures made out of low-carbon steel [18]. Further, in order to generate the data for $\Gamma_{h}^{*}$ as a function of $H^{*}$, the following values are used: $R e_{l}=20$ (as operating condition), $\varepsilon=0.3, K^{*}=10^{-8}, n=3$ (as properties of the medium), $m_{\phi l}=m_{\phi v}=4, m_{s l}=m_{s v}=0.25, \psi_{l}=$ $\psi_{v}=0.75, \Delta T_{l}=\Delta T_{v}=0.5{ }^{\circ} \mathrm{C}, s_{l}=0.995$ and $s_{v}=0.005$ (as adjustable smoothing parameters).

Fig. 2 clearly shows that the effective diffusion coefficient, other than being a strong function of $H^{*}$, is discontinuous at saturated liquid and vapor conditions. It is obvious that the application of smoothing algorithm, suggested by Alomar et al. [14] restricts the effective diffusivity to finite values, i.e., preventing it from going to zero, at $s=0$ and $s=1$ in the two-phase region. It may be further noted that the drastic change in $\Gamma_{h}^{*}$ is more prominent close to the saturated vapor condition, even after employing the proposed smoothing algorithm; perhaps explaining the reason for not using the TPMM of Wang [8] for the simulation of phase change process inside porous media from the two-phase mixture to the superheated vapor state.

TABLE IV: THERMO-PHYSICAL PROPERTIES OF WATER

\begin{tabular}{lcc}
\hline \hline Property & Liquid & Vapor \\
\hline Density, $\rho\left(\mathrm{kg} / \mathrm{m}^{3}\right)$ & 957.85 & 0.5978 \\
Specific heat, $C_{p}(\mathrm{~J} / \mathrm{kgK})$ & 4190.2 & 2029.0 \\
Dynamic viscosity, $\mu(\mathrm{kg} / \mathrm{ms})$ & $2.79 \times 10^{-4}$ & $1.202 \times 10^{-5}$ \\
Thermal conductivity, $\mathrm{k}(\mathrm{W} / \mathrm{mK})$ & 0.68 & 0.0248 \\
Saturation enthalpy, $h(\mathrm{~J} / \mathrm{kg})$ & $419.02 \times 10^{3}$ & $2676.05 \times 10^{3}$ \\
Latent heat of evaporation, $h_{f g}(\mathrm{~J} / \mathrm{kg})$ & $2257.03 \times 10^{3}$ \\
Surface tension coefficient, $\sigma(\mathrm{N} / \mathrm{m})$ & \multicolumn{2}{c}{0.0589} \\
\hline \hline
\end{tabular}

\section{RESUlTS AND Discussion}

\section{A. Ranges of Parameters}

Simulations of complete phase change process within a divergent porous evaporator is carried out during the present investigation by applying the new smoothing strategy for effective diffusion coefficient, as briefly explained in the foregoing section, in order to avoid "jump" in the predicted temperature profile that may occur at the interfaces of two-phase regions. For all the cases presented in this article, $R_{i}$ is taken as $25 \mathrm{~mm}, R_{o}$ is varied between $28 \mathrm{~mm}$ and $35 \mathrm{~mm}$ and $L$ is varied from $375 \mathrm{~mm}$ to $425 \mathrm{~mm}$. The length of the divergent section $l_{D}$ depends on $l_{i}$ and $l_{o}$. In the present study, $l_{i}$ is varied between $0.1 L$ and $0.2 L$, whereas $l_{o}$ is kept fixed at $0.1 L$. Entire duct is filled up with a porous medium for which the porosity is varied between 0.2 and 0.4 and the permeability is changed from $6.25 \times 10^{-11}$ to $6.25 \times 10^{-13}$.

Liquid water at $T_{i n}=20^{\circ} \mathrm{C}$ enters the duct which is then heated to the superheated vapor state by adding constant heat flux, ranging from $18 \mathrm{~kW} / \mathrm{m}^{2}$ to $24.5 \mathrm{~kW} / \mathrm{m}^{2}$, at the periphery of divergent section of the duct. In a typical application involving phase change inside porous media, the mass flow rate of water is generally quite low and hence it is varied between $1.25 \mathrm{~kg} / \mathrm{hr}$ and $2 \mathrm{~kg} / \mathrm{hr}$, allowing the average inlet velocity is to vary from $0.2 \mathrm{~mm} / \mathrm{s}$ to $0.25 \mathrm{~mm} / \mathrm{s}$. Therefore, in the present study, the following ranges of dimensionless parameters are considered:

Porosity: $\varepsilon=0.2-0.4$.

Darcy number: $K^{*}=10^{-7}-10^{-9}$

Inlet Reynolds number: $R e_{l}=17.5-22.5$

Inlet radius: $R_{i}^{*}=1$; Outlet radius: $R_{o}^{*}=1.12-1.4$

Total length: $L^{*}=15-17$

Inlet length: $l_{i}=0.1 L-0.2 L$; Outlet length $l_{o}=0.1 L$

Length of divergent section: $l_{D}=0.7 \mathrm{~L}-0.8 \mathrm{~L}$

Heat Flux: $\dot{Q}_{w}^{\prime \prime *}=4 \times 10^{-2}-4.5 \times 10^{-2}$,

where, the reference case is taken for $\varepsilon=0.3, K^{*}=10^{-8}$, $R e_{l}=20, R_{o}^{*}=1.26, L^{*}=16, l_{i}=0.1 L, l_{o}=0.1 L, l_{D}=0.8 L$, and $\dot{Q}_{w}^{\prime *}=4.25 \times 10^{-2}$ and hence these parameters are kept fixed for all the cases, unless otherwise mentioned. They are varied only when their effects on phase change is studied.

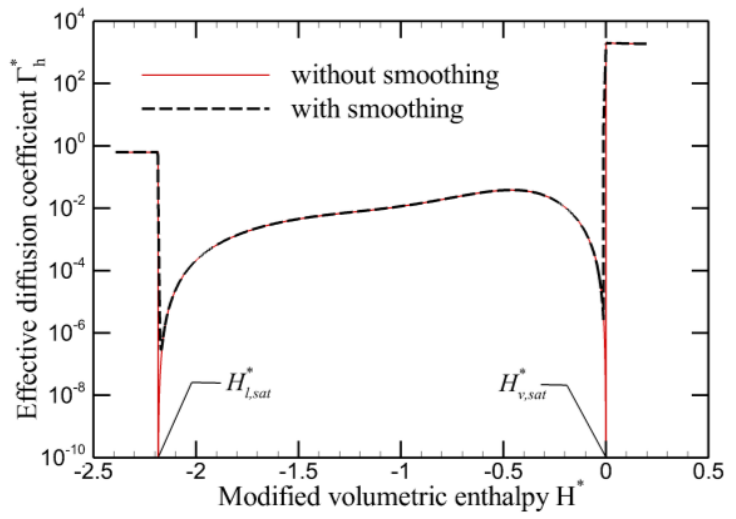

Fig. 2. Comparison of effective diffusion coefficient with and without smoothing treatment at $\varepsilon=0.3$ and $K^{*}=10^{-8}$.

\section{B. Consequence of Smoothing of Diffusion Coefficient}

Prior to obtaining results for the present investigation, a detailed grid independence study is carried out and it is observed that 1000 uniform control volumes are required for obtaining grid-independent results. In order to establish the usefulness of proposed smoothing algorithm for effective diffusion coefficient, results obtained in terms of the predicted axial temperature with and without its application are first presented in Fig. 3, where (a) shows the variation for different porosities with a low heat flux $\dot{Q}_{w}^{\prime \prime^{*}}=3 \times 10^{-2}$ condition, while in (b) the effect of variable exit radius (divergence angle) is presented for the base heat flux of $\dot{Q}_{w}^{\prime *}=4.25 \times 10^{-2}$. It is evident from the figure that when phase change takes place only from the sub-cooled liquid to the two-phase mixture, no "jump" in the predicted temperature, close to the saturated liquid condition, is observed and solutions, obtained with and without the smoothing algorithm, are identical. On the other hand, for higher heat flux condition, 
when phase change also takes place from the two-phase mixture to the superheated vapor, discontinuous temperature profiles are predicted if the proposed smoothing algorithm is not applied. Nevertheless, even for this case, the comparison also clearly shows that both solutions match each other close to the evaporator exit, indicating that the global energy balance is satisfied by both methods.

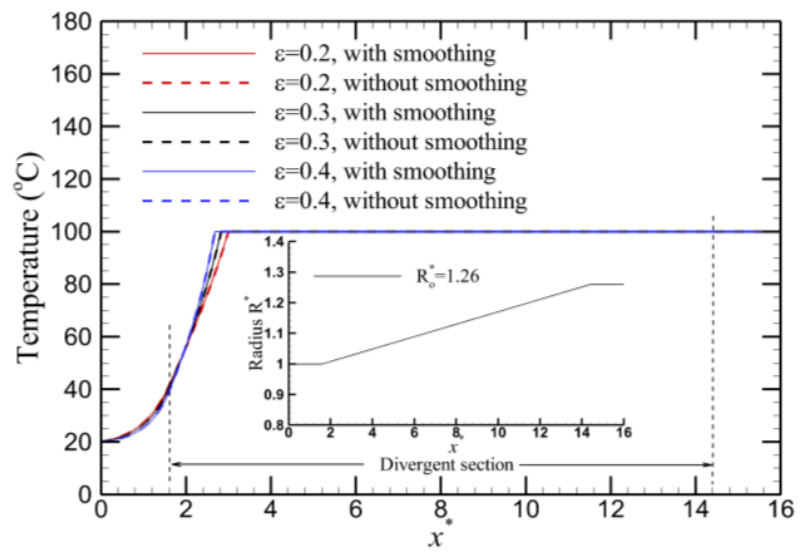

(a) Incomplete phase change

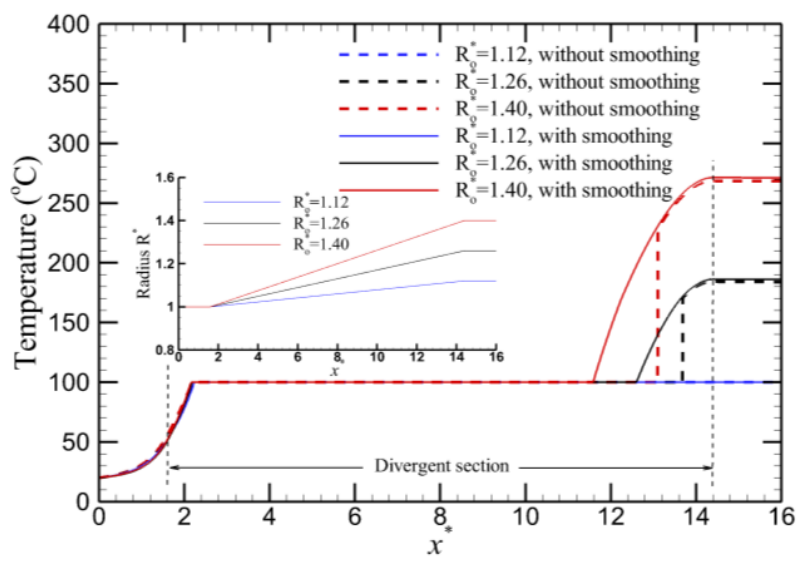

(b) Complete phase change

Fig. 3. Comparison of predicted temperature profiles with and without applying smoothing algorithm: (a) partial phase change for different porosities with $\dot{Q}_{w}^{\prime *}=3 \times 10^{-2}$, (b) complete phase change for different divergence angles with $\dot{Q}_{w}^{\prime *}=4.25 \times 10^{-2}$.

It may be noted here that the energy conservation equation is strongly nonlinear in nature. During discretisation, the nonlinearity is addressed in a semi-implicit manner, i.e., by pretending the coefficients, those depend on $H^{*}$, to be known from the previous iteration. As a result, the rate of convergence as well as converged solutions for certain cases depend on the guessed values. It is observed that if the converged solution obtained with smoothing is used as a guessed solution to the solver that works without smoothing, the latter code not only converges very fast (since guessed values are too close to the true solution), but also to the nearly identical solution without any "jump" in predicted properties. Since these new solutions cannot be separately distinguished from those obtained using the smoothing algorithm, they could not be shown in Fig. 3. It can, therefore, be safely concluded that the proposed smoothing algorithm provides a successful remedy for the occurrence of "jump" in predicted properties without modifying the true (expected) solution and hence can be recommended for the simulation of complete phase change process within porous media.

\section{Effects of Operating Conditions}

Effects of heat addition and inlet Reynolds number on predicted temperature distributions are shown in Fig. 4. As a general observation, it may be recognized that for the present problem, heat is added only in the diffuser section (marked as the "divergent section" in all similar figures) while the pipe surface at inlet and exit is kept insulated. One may, therefore, expect the temperature at inlet and exit sections to remain unchanged, with their values being equal to those at inlet and exit of the evaporator, respectively. Although this is somewhat true for the exit section, the figure (for that matter all similar figures) clearly shows an increase in fluid temperature at the inlet section. This observation, however, is expected since the phase change problem inside porous media being elliptic in nature, axial diffusion plays a significant role which heats up the upstream section. Most importantly, owing to features of present formulation, axial diffusion remains present even in the two-phase region as there exists a finite gradient of $H^{*}$, although the temperature remains unchanged at saturation condition. Since heat is added in the backward direction due to axial diffusion, no back-heating effect is felt at the exit section, whereas it is quite prominent at the inlet section which is followed by the heating section.

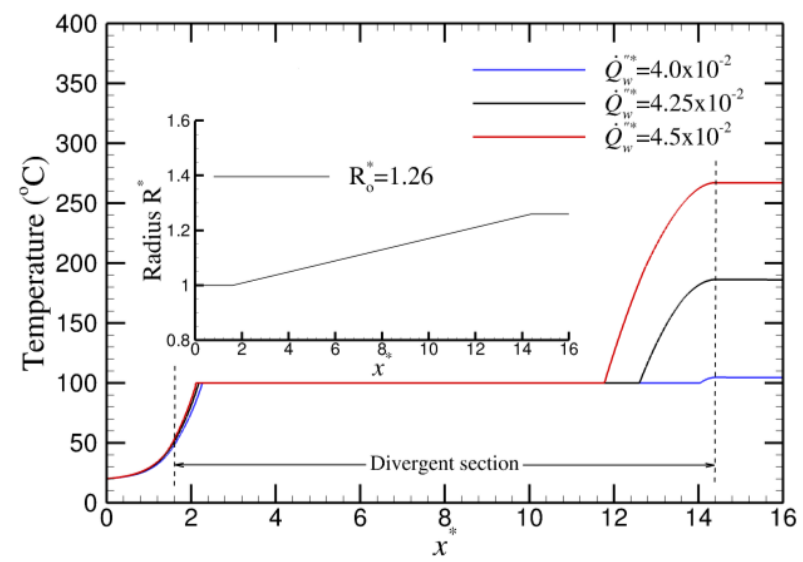

(a) Effect of applied heat flux

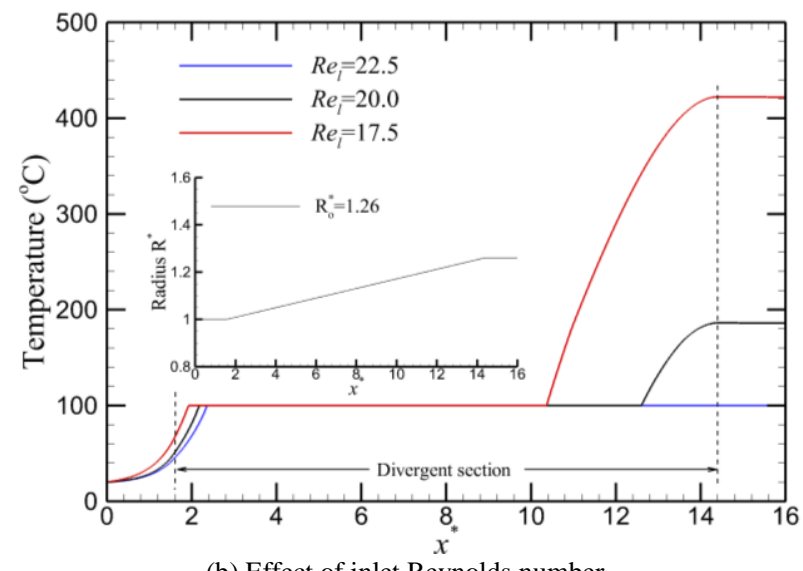

(b) Effect of inlet Reynolds number.

Fig. 4. Effect of (a) applied heat flux and (b) inlet Reynolds number.

Other than this general observation, the effect of heat flux is more evident in the vapor phase and is less prominent for the liquid phase, as may be seen from Fig. 4(a). On the other hand, Fig. 4(b) clearly shows that reduction of inlet Reynolds 
number (i.e., decrease in mass flow rate and hence increase in residence time) leads to higher exit temperature. Although both these observations are quite expected, Fig. 4 clearly indicates that a marginal change in the operating condition can lead to a substantial change in the exit temperature. Therefore, operating conditions are required to be properly designed in order to achieve the desired objective.

\section{Effects of Porous Media Properties}

Fig. 5 illustrates the effects of porous media properties (in the form of porosity and permeability) on temperature distribution within the porous evaporator. In order to study these effects, the porosity is changed by two-folds (from 0.2 to 0.4 ), whereas the Darcy number is changed by two orders of magnitude (from $10^{-9}$ to $10^{-7}$ ). Perhaps this explains more pronounced effect of permeability (see Fig. 5b), as compared to porosity (see Fig. 5a), on the temperature distribution.

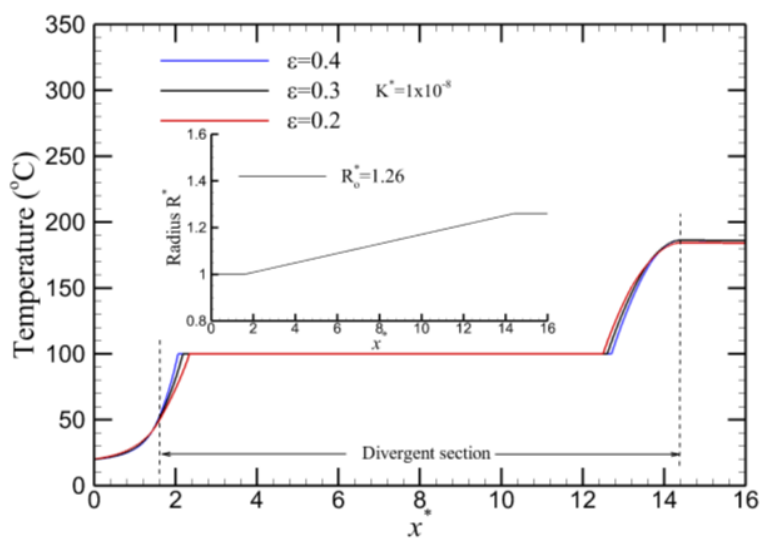

(a) Effect of porosity

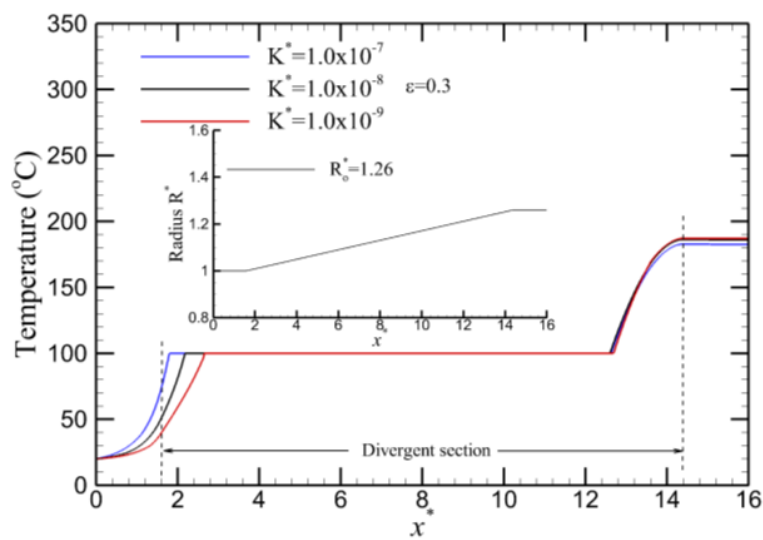

(b) Effect of Permeability (Darcy number)

Fig. 5. Effect of (a) porosity and (b) permeability (Darcy number).

As far as the energy transport during phase change process is concerned, porous media properties affect the temperature distribution by modifying the effective diffusion coefficient, which changes the amount of energy transport in the upstream direction due to axial diffusion. As evident from Table II, an increase in porosity reduces $\Gamma_{h}$ in the single phase region, by reducing the contribution of solid-phase conductivity in $k_{\text {eff }}$, whereas it enhances $\Gamma_{h}$ by increasing $D$ in the two-phase region. As a result, phase change initiates early with the increase in porosity and the process is prolonged to a larger axial distance. On the other hand, an increase in permeability increases $\Gamma_{h}$ only in the two-phase region by enhancing the value of $D$. This causes early initiation of the phase change process and exit solutions remain nearly unaffected.

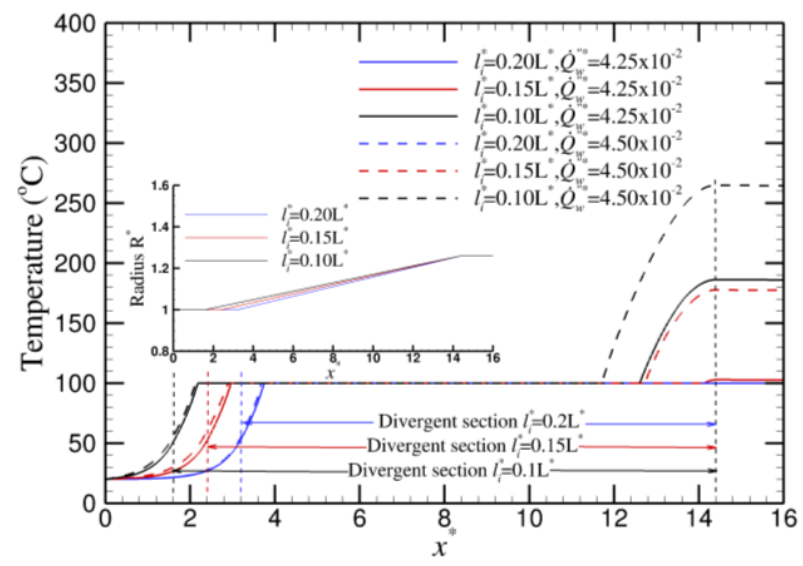

(a) Effect of inlet length

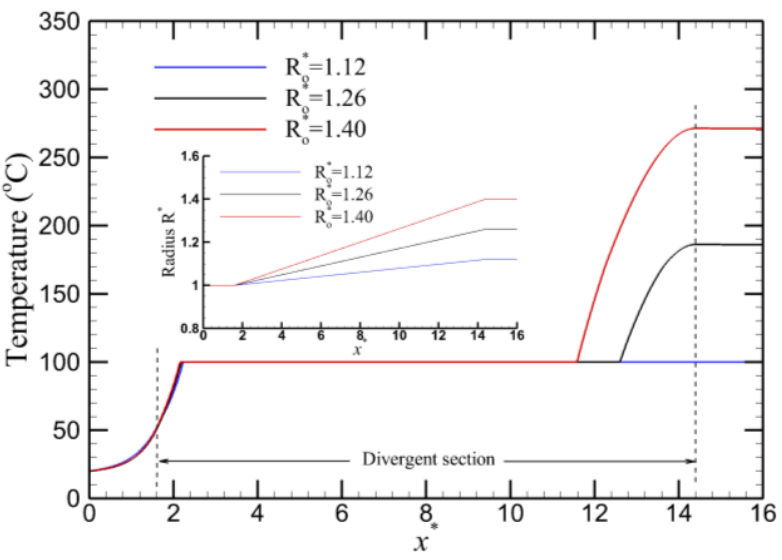

(b) Effect of radius ratio

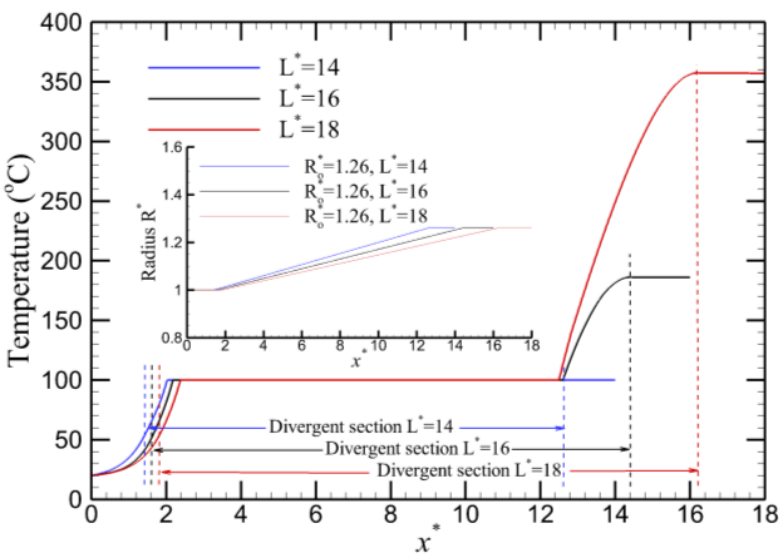

(c) Effect of evaporator length

Fig. 6. Effect of (a) inlet length (b) diffuser angle and (c) evaporator length.

\section{E. Effects of Evaporator Geometry}

Effects of diffuser geometry, in terms of inlet length, radius ratio and length of the evaporator, on the temperature distribution are summarized in Fig. 6. It may be recognized here that the decrease in inlet length, the increase in diffuser angle (i.e., outlet diameter) and the increase in total length of the diffuser effectively enhance the surface area where heat flux is actually added. With the increase in heated surface area by keeping the same heat flux, the total heat input to the working fluid increases, which in turn, enhances the exit temperature of fluid from the evaporator. It is also evident from Fig. 6 that the exit quality of steam is extremely sensitive to the geometry of the divergent porous evaporator and hence 
adequate care must be taken while designing such evaporators in order to meet the process requirement.

\section{CONCLUSIONS}

In the present study, a thorough numerical investigation is performed for complete phase change process within a divergent porous evaporator. A newly proposed smoothing algorithm [14] is adopted in order to deal with the discontinuity in the effective diffusion coefficient, which proves to be essential for successfully avoiding the occurrence "jump" in the predicted temperature. Present results clearly indicate that operating conditions and the geometry of diffuser strongly affect the outlet condition of steam, whereas, porous media properties have only minor influence. Most importantly, the analysis, presented in this article, provides a useful tool for designing a divergent porous evaporator in order to achieve complete phase change.

\section{REFERENCES}

[1] C.Y. Wang and P. Cheng, "Multiphase flow and heat transfer in porous media," Advances in Heat Transfer, Academic Press, New York, vol. 30, 1997, pp. 93-196.

[2] A. E. Scheidegger, The Physics of Flow through Porous Media, $3^{\text {rd }}$ ed. University of Toronto Press, Toronto 1974.

[3] J. Bear, Dynamics of Fluids in Porous Media, New York, Elsevier, 1972.

[4] P. S. Ramesh and K. E. Torrance, "Numerical algorithm for problems involving boiling and natural convection in porous materials," Numer. Heat Transfer, Part B, vol. 17, no. 1, pp. 1-24, January 1990.

[5] P. S. Ramesh and K. E. Torrance, "Boiling in a porous layer heated from below: effects of natural convection and a moving liquid two-phase interface," J. Fluid Mech., vol. 27, pp. 289-309, May 1993

[6] C. Y. Wang and C. Beckermann, "A two-phase mixture model of liquid-gas flow and heat transfer in capillary porous media, I. formulation," Int. J. Heat Mass Transfer, vol. 36, no. 11, pp. 2747-2758, July 1993.

[7] C. Y. Wang, C. Beckermann, and C. Fan, "Numerical study of boiling and natural convection in capillary porous media using the two-phase mixture model," Numer. Heat Transfer, Part A, vol. 26, pp. 375-398, October 1994.

[8] C. Y. Wang, "A fixed-grid numerical algorithm for two-phase flow and heat transfer in porous media," Numer. Heat Transfer, Part B, vol. 32 , pp. 85-105, February 1997.

[9] T. S. Zhao, P. Cheng, C. Y. Wang, "Buoyancy-induced flows and phase-change heat transfer in a vertical capillary structure with symmetric heating," Chem. Eng. Sci., vol. 55, pp. 2653-2661, April 2000.

[10] M. Najjari and S. B. Nasrallah, "Numerical study of boiling in inclined porous layer," J. Porous Media, vol. 6, no. 1, pp. 71-81, 2003.

[11] M. Najjari and S. B. Nasrallah, "Heat transfer between a porous layer and a forced flow: Influence of layer thickness," Drying Technol, vol. 27, pp. 336-343, 2009.

[12] H. Y. Li, K. C. Leong, L.W. Jin, and J. C. Chai, "Transient two-phase flow and heat transfer with localized heating in graphite foams," Int. J. Therm. Sci., vol. 49, pp. 1115-1127, July 2010.

[13] H. Y. Li, K. C. Leong, L.W. Jin, and J. C. Chai, "Transient behavior of fluid flow and transfer with phase change in vertical porous media," Int. J. Heat Mass Transfer, vol. 53, pp. 5209-5222, November 2010.

[14] O. R. Alomar, M. A. A. Mendes, D. Trimis, and S. Ray, "Numerical simulation of complete liquid-vapor phase change phase change process inside porous media using smoothing of diffusion coefficient," Int. J. of Thermal Science, submitted for publication, 2014.

[15] S. V. Patankar, Numerical Heat Transfer and Fluid Flow, New York, Hemisphere, 1980

[16] S. W. Churchill and R. Usagi, "A general expression for the correlation of rates of transfer and other phenomena," AIChE. J., vol. 18, pp. 1121-1128, November 1972.

[17] F. P. Incropera, D. P. Dewitt, T. L. Bergman, and A. S. Lavine, Fundamentals of Heat and Mass Transfer, John Wiley \& Sons, 2007.
[18] C. Borgnakke and R. E. Sonntag, Fundamentals of Thermodynamics, John Wiley \& Sons, 2009.

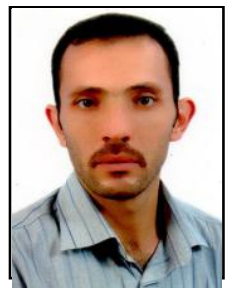

Omar Rafae Alomar was born in Mosul, Iraq, 1979. $\mathrm{He}$ received the B.Sc. and M.Sc. degrees in mechanical engineering from the Mosul University, Mosul, Iraq, in 2001 and 2004, respectively.

From 2005 till date, he is working in the Refrigeration and Air Conditioning Technologies Engineering Department, Technical College of Mosul, Mosul, Iraq as an assistant lecturer. From 2011, he is pursuing Ph.D. degree at the Institute of Thermal Engineering (IWTT), Technische Universität Bergakademie Freiberg (TU-BAF), Germany, with DAAD fellowship. He has experience in CFD, phase change heat transfer, natural and forced convection within porous media, internal combustion engines, refrigeration and air conditioning systems.

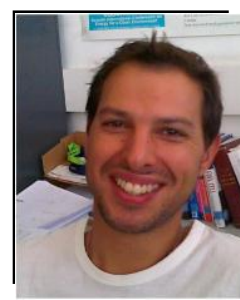

Miguel Abreu Almeida Mendes was born in Lisbon, Portugal, 1981. He received the M.Sc. and Ph.D degrees in mechanical engineering from the Institutor Superior Tecnico, Lisbon, Portugal, in 2005 and 2011 , respectively.

$\mathrm{He}$ is working as a post-doctoral researcher at the IWTT, TU-BAF, Germany, from September 2011, where he is leading the subproject $\mathrm{B}$, which is a part of the Collaborative Research Center CRC 920, funded by the German Research Foundation (DFG). He has experience in numerical methods, computational fluid dynamics and modeling of energy conversion systems, in particular involving porous media and combustion.

Dr. Mendes published several articles in international journals and also acts as the reviewer for many of them.

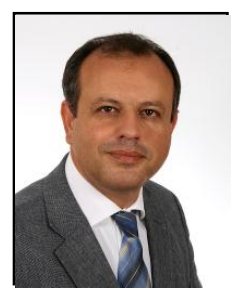

Dimosthenis Trimis was born in Athens, Greece, 1967. He received the Dipl.-Ing. (M.Sc.) degree in mechanical engineering from the NTUA, Athens, Greece, in 1990 and obtained his PhD (Dr.-Ing.) degree from the Friedrich Alexander Universität Erlangen, Nürnberg, Germany, in 1995.

From 2006 till August 2013, he worked as the chair of Gas and Heat Technology at IWTT, TU-BAF, Germany. Since September 2013, he is working as the Honorary Professor at the same chair and at the same time he has taken up the responsibility as the Head, Chair for Combustion Technology, Karlsruhe Institute of Technology, Germany. His research interests are in the fields of combustion technology, partial oxidation, porous media, energy, etc.

Prof. Trimis has substantial number of publications in international journals and conferences. He has received the Italgas Research Price in 1998 and the Wilhelm-Jost-Medal of the German section of the Combustion Institute in 2001. Other than being member of several professional bodies, Prof. Trimis acts as the reviewer for many international journals and funding agencies, like DFG and European Union.

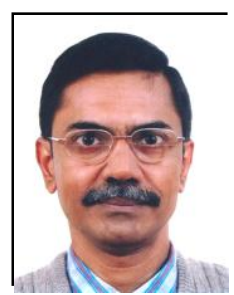

Subhashis Ray was born in Kolkata, India, 1965. He received his B.M.E in 1987 from Jadavpur University (JU), Kolkata, India, M.E. in 1989 from the Indian Institute of Science, Bangalore, in mechanical engineering and thermal sciences, respectively. In 1999, he obtained his Ph.D. in engineering from the Indian Institute of Technology, Bombay.

From January 1990 till June 2007 he worked in the Department of Mechanical Engineering, JU, as a faculty member in various capacities. Since July 2007, he has taken up the responsibility as the head, Theory and Numerical Simulations Group and from June 2012, he is working as a professor, Modelling of Phase Change Processes and Heat Exchangers at the IWTT, TU-BAF, Germany. His research interests are in the fields of computational fluid dynamics, phase change processes, porous media, heat and mass transfer, etc.

Prof. Ray has substantial number of publications in international journals and conferences and he acts as the reviewer for many of them. He is the recipient of several scholarships including the Alexander von Humboldt fellowship for post-doctoral research in Germany. He is also member of several professional bodies. 